\title{
Haldane's Fractional Statistics and the Lowest Landau Level on a Torus
}

\author{
Ansar Fayyazuddin ${ }^{1}$ and Dingping $\mathrm{Li}^{2,3}$ \\ ${ }^{1}$ NORDITA, Blegdamsvej 17, DK-2100 Copenhagen Ø, Denmark \\ ${ }^{2}$ Dipartimento di Fisica and Sezione I.N.F.N., Universitá di Perugia, Via A. Pascoli, I-06100 Perugia, Italy \\ ${ }^{3}$ Division de Physique Théorique, IPN, Orsay Fr-91406, France
}

(September 2, 2018)

\begin{abstract}
The lowest Landau level on a torus is studied. The dimension of the many-body Hilbert space is obtained and is found to be different from the formula given by Haldane. Our result can be tested in numerical investigations of the low-energy spectrum of fractional quantum Hall states on a torus.
\end{abstract}

PACS numbers: 73.20.Dx, 03.65.Fd, 05.30.-d, 73.20.mf.

Identical particles with statistics interpolating between Bose and Fermi can exist in two dimensions [1]. Particles which obey fractional statistics (neither bosonic nor fermionic) are called anyons. Anyon physics has been applied to the theory of the fractional quantum Hall effect (FQHE) 2, 3. The condensation of the anyonic quasiparticles [4.5] in Laughlin states gives rise to hierarchical states in the FQHE [4, 6].

Recently, Haldane proposed [7] a new definition of fractional statistics by generalizing the Pauli exclusion principle (we will call it Haldane's statistics or HS). The definition of HS is based on a Hilbert space counting argument (see Refs. 8 10] for further developments). In the framework of HS the monodromy properties of wavefunctions (which are usually used in the definition of statistics) are not used to define the statistics and the definition of statistics is independent of the dimension of space.

Following [7], we consider a $N$ body system in a finite space, and fix $N-1$ particles among them. Analyze the Hamiltonian of the remaining particle assuming that the dimension $d$ of the Hilbert space for this particle is finite and independent of which particle has been chosen. The parameter $g$ of HS is by definition

$$
\Delta d=-g \Delta N .
$$

For bosons, $g=0$, and for fermions, $g=1$. For $g$ neither 0 nor 1, we say that particles obey fractional statistics. If Eq. (11) is satisfied, we say that particles satisfy HS. The size of the Hilbert space of the many-body system at fixed $N$ has been argued to be equal to [7]

$$
\frac{(d+N-1) !}{(N) !(d-1) !} .
$$

One of the advantages of the concept of HS is that for bosons or fermions the number of N-body states among accessible states is given by the unified Eq. (2). In various examples of HS, for example, anyons in a very strong magnetic field on a sphere, Haldane-Shastry Spin Chain model etc., Eq. (2) was found to be satisfied. One would guess it may be true in any example of HS.

In Refs. [11,12], the emphasis was put on the Hilbert space of the low-energy sector of fractional quantum Hall $(\mathrm{FQH})$ states on a sphere, in the presence of quasielectron
(QE) or quasihole (QH) excitations. On the other hand, the energy spectrum of a few electrons can be calculated numerically. A low-energy sector was found which is well separated from the groundstate and corresponds to Hall states with QE or QH excitations. The dimension of the Hilbert space of such states predicted in Ref. []] by using Eq. (2), for example, in the presence of $\mathrm{QH}$ excitations, is $\left(N_{e}+N_{q}\right) ! /\left[\left(N_{e}\right) !\left(N_{q}\right) !\right]$, which is in agreement with the number of states in the low-energy sector (NSLES) found in numerical studies. Furthermore, anyons on the sphere with hard core boundary conditions interacting with a strong magnetic field were considered in [13], and the dimension of the Hilbert space of many-body states (DHMS) was found to be given by Eq. (2), in agreement with 11. 12.

In this letter, we consider anyons in a strong magnetic field on a torus, thus only the lowest Landau level (LLL) states. We explicitly work out the formula of the DHMS, which is the number of possible many-body states in the LLL. We note that the concept of HS can also be applied to our case and expect the formula of the DHMS to be given by Eq. (2) as in the case of spherical geometry. However, the DHMS found in this case is not given by Eq. (2). By using the results of this letter we also obtain the NSLES of FQH states on a torus by using Halperin's theory of hierarchical states. Our formula can be tested in numerical calculations on a torus, similar to Ref. 12] where the calculation was performed on a sphere. We conclude that when the topology is changed to that of a torus, the prediction made by HS does not tally with what we find for anyons in the LLL on a torus. One may guess that Eq. (2) is valid only when there are no topological obstructions for constructing single component wavefunctions. The nontrivial topology of tori forces the wavefunctions of anyons to be multi-component, in contrast to the case of spheres [14 18].

The NSLES of FQH states on a sphere was obtained in Jain's composite fermion theory of the FQHE [19], and the result was found to be identical to the one predicted in Halperin's hierarchical theory using the HS concept. However we do not know how to use Jain's theory to obtain the NSLES of FQH states on a torus because of difficulties in the construction of Jain's wavefunctions on 
a torus with the correct degeneracy. FQH states have degenerate ground states on a torus [20], which can be seen in numerical calculations 21]. We note that one can construct Halperin's hierarchical wavefunctions on a torus with the correct degeneracy [22].

We begin by solving the DHMS of anyons in the LLL on a torus at a specific filling. Following the notation in Ref. [22], the Hamiltonian of a quasihole in singular gauge [16] 18] is $H=\frac{1}{2 m}\left[\left(p_{x}-e_{q} B y\right)^{2}+\left(p_{y}\right)^{2}\right]$, where $B$ is the magnetic field and $e_{q}$ is the charge of the particle. Let $\Phi$ be the magnetic flux seen by the particle, $\Phi=\tau_{2} e_{q} B$. We take the statistical parameter $\pi \alpha=\pi k / s$, where $k, s$ are positive coprime integers. The statistical phase is then $e^{i \alpha \pi}$. $\alpha$ must be a rational number on a torus and $\Phi-\alpha N=\Phi^{\prime}$ must be an integer according to the Dirac quantization condition. Thus we are considering the case where the statistical flux is opposed to the flux of the external magnetic field. The situation where the two fluxes are parallel with hard core boundary conditions imposed as in Refs. 111 13 will be considered in detail elsewhere and a short discussion of such a situation in the case of QE excitations can be found at the end of this letter. Although it is usual to restrict $|\alpha|<1$ because the statistical phase $e^{i \alpha \pi}$ depends only on $\alpha \bmod 2$, we allow $\alpha>0$ to be unrestricted as in Ref. 12. Here $\alpha$ is related to the amount of statistical flux bound to each particle. The wavefunctions are represented by $s$-component vectors. In our case, the wavefunctions can be written as

$\Psi\left(z_{i}\right)_{n}=\exp \left(-\frac{\pi \Phi \sum_{i} y_{i}^{2}}{\tau_{2}}\right) \prod_{i<j}\left[\theta_{3}\left(z_{i}-z_{j} \mid \tau\right)\right]^{k / s} F\left(z_{i}\right)_{n}$

where $n=1, \cdots, s$ is the component index of the wavefunctions (all numbers appearing above and below are positive if not otherwise specified).

We can separate the center of mass coordinate part of the wavefunctions from the relative part in the case of $\Phi^{\prime}=p N$ where $p$ is an integer by generalizing the arguments of Ref. [23]. As $\Phi / N=(k / s)+p$, the functions $F\left(z_{i}\right)_{n}$ are given as

$$
F\left(z_{i}\right)_{n}=\theta\left[\begin{array}{c}
a_{m, n} \\
b
\end{array}\right]\left(\sum_{i} z_{i} e_{2} \mid e_{1}, \tau\right) F^{\prime} .
$$

$F^{\prime}$ depends on the relative holomorphic coordinates $\left(z_{i}-\right.$ $z_{j}$ ) and satisfies certain translational properties around the handles (or the nontrivial homology cycles) on the torus. In Eq. (4), the notation is

$$
\begin{aligned}
& \left(e_{1}\right)^{2}=s(k+p s),\left(e_{2}\right)^{2}=p+(k / s), \\
& a_{m, n}=a_{m, n}^{*} e_{1}^{*}, b=b^{*} e_{1}^{*} ; e_{1}^{*}=\frac{1}{e_{1}}, e_{2}^{*}=\frac{1}{e_{2}}, \\
& a_{m, n}^{*}=a_{0}+m s+n(k+p s), b_{i}^{*}=b_{0}, \\
& m=1, \cdots, k+p s, n=1, \cdots, s
\end{aligned}
$$

$a_{0}, b_{0}$ are fixed by boundary conditions and $m$ is the index of the center-coordinate degeneracy (CCD). When $p$ is an even integer, for the wavefunctions of Laughlin type, $F^{\prime}$ is equal to $F^{\prime}(p)_{l a u}=\prod_{i<j}\left[\theta_{3}\left(z_{i}-z_{j} \mid \tau\right)\right]^{p}$. therefore in this case, the degeneracy of the wavefunctions is equal to the CCD: $k+p s$ 22.

To obtain the DHMS of the model when $\Phi^{\prime}=p N$ and $p$ is an even integer, we find the number of all possible wavefunctions, or possible $F^{\prime}$ (or possible $F_{n}$ ), not necessarily restricted to be of Laughlin type. The wavefunctions of the center coordinate part shall remain unchanged with respect to different solutions of $F^{\prime}$ [23].

To work out $D_{r}$, the number of all possible $F^{\prime}$, it is not necessary to get explicit solutions for all possible $F^{\prime}$. Considering $N$ bosonic particles and taking the magnetic flux quanta $\Phi$ equal to $\Phi_{p}=p N$, the wavefunctions of this model are given by Eq. (4), with $\Phi=p N$, $\left(e_{1}\right)^{2}=p,\left(e_{2}\right)^{2}=1, \alpha=0$ and $m=1, \cdots, p, n=1$. One can show that $F_{b o}^{\prime}$, the wavefunction of the relative part in the bosonic model, satisfies the same translational properties and has the same dependence on the relative holomorphic coordinates as $F^{\prime}$ in the anyon model. Thus we have the same solutions for $F^{\prime}$ and $F_{b o}^{\prime}$ according to Algebraic Geometry, this implies that the number of possible $F^{\prime}, D_{r}$, is equal to the number of possible $F_{b o}^{\prime}$. The DHMS (we recall that the DHMS is the number of all possible states) of the bosonic model is $D_{b o}=\left(\Phi_{p}+N-1\right) ! /\left[\left(\Phi_{p}-1\right) !(N) !\right]$ because the number of possible states for a single particle is equal to $\Phi_{p}$. Therefore $D_{r}$ is equal to $D_{b o}$ divided by the CCD of the wavefunctions, which is equal to $p$ here. This leads to $D_{r}=(1 / p) D_{b o}=\left(\Phi_{p}+N-1\right) ! /\left[\left(\Phi_{p}\right) !(N-1) !\right]$. Thus the DHMS of the anyon model is equal to $D=D_{r} \times(k+$ $p s)$ where $(k+p s)$ is the CCD of the model. By using the relation $\Phi^{\prime}=\Phi_{p}=p N$, we have $N(k+p s)=N k+\Phi^{\prime} s$. Inserting this relation in the formula for $D$ we obtained above, $D$ can be rewritten as

$$
D=\left(N k+\Phi^{\prime} s\right) \frac{\left(\Phi^{\prime}+N-1\right) !}{\left(\Phi^{\prime}\right) !(N) !} .
$$

In the discussion above we obtained the DHMS of anyons in the LLL on a torus when $\Phi^{\prime}=\Phi_{p}=p N$ and $p$ is an even integer. Eq. (6) depends only on $\Phi^{\prime}, \alpha(\alpha=$ $k / s)$ or $\Phi$ and $\alpha$. There is no explicit dependence on the parameter $p$ in the final formula although we used the relation $\Phi^{\prime}=p N$ in the derivation of the formula.

We now generalize the discussion to the case of arbitrary integer $\Phi^{\prime}$ and show that formula (6) still holds. For any integer $\Phi^{\prime}$, one can write $\Phi^{\prime}=\left[k^{\prime} / s^{\prime}\right] N$ where $k^{\prime}, s^{\prime}$ are coprime integers.

We first consider $\mathrm{N}$ bosonic particles with flux $\Phi_{b o}=$ $\Phi^{\prime}=\left[k^{\prime} / s^{\prime}\right] N$. The wavefunctions are given by Eq. (3) with $\Phi=\Phi_{b o}$. The wavefunctions of the center coordinate part in $F\left(z_{i}\right)_{b o}$ are given by $\theta\left[\begin{array}{c}a_{m, l} \\ b\end{array}\right]\left(\sum_{i} z_{i} e_{2} \mid e_{1}, \tau\right)$ (these can be obtained, for example, by following Ref. 23), where $\left(e_{1}\right)^{2}=k^{\prime} s^{\prime},\left(e_{2}\right)^{2}=\left(k^{\prime} / s^{\prime}\right), a_{m, l}^{*}=a_{0}+$ $m s^{\prime}+l k^{\prime}$ and $m=1, \cdots, k^{\prime}, l=1, \cdots, s^{\prime}$. The CCD of 
the model is equal to $k^{\prime}$. Because $l$ is not restricted to be one, when one coordinate is moved around the handles, the index $l$ will be changed. One may say that the wavefunctions are multi-component. However, this cannot be correct as the wavefunctions of bosonic and fermionic particles are single-component. This apparent paradox arises due to the fact that factorization of the wavefunction into relative and center parts is possible only when $\Phi / N$ is an integer due to obstructions caused by translation properties of the wavefunctions. In the present situation, $F\left(z_{i}\right)_{b o}$ should be expanded as $\sum_{l} \theta\left[\begin{array}{c}a_{m, l} \\ b\end{array}\right]\left(\sum_{i} z_{i} e_{2} \mid e_{1}, \tau\right) F_{l}^{\prime b o}$. The wavefunctions of the relative part (denoted as $F_{l}^{\prime b o}$ here ) are also multicomponents and satisfy certain translational properties. When one coordinate is moved around the handles, the index $l$ inside the summation will be changed to another number. But the final wavefunctions are singlecomponent due to the contraction of the index $l$.

Now we consider the anyon model with $\Phi^{\prime}=\left(k^{\prime} / s^{\prime}\right) N$. As $\Phi / N=(k / s)+\left(k^{\prime} / s^{\prime}\right)=\left(k s^{\prime}+s k^{\prime}\right) /\left(s s^{\prime}\right)$, the wavefunctions of the center coordinate part in $F\left(z_{i}\right)$ are given by the functions $\theta\left[\begin{array}{c}a_{m, n, l} \\ b\end{array}\right]\left(\sum_{i} z_{i} e_{2} \mid e_{1}, \tau\right)$ with $\left(e_{1}\right)^{2}=s^{\prime} s\left(k s^{\prime}+s k^{\prime}\right),\left(e_{2}\right)^{2}=(k / s)+\left(k^{\prime} / s^{\prime}\right), a_{m, n, l}^{*}=$ $a_{0}+m s s^{\prime}+(l s+n)\left(k s^{\prime}+s k^{\prime}\right)$ and $m=1, \cdots,\left(k s^{\prime}+\right.$ $\left.s k^{\prime}\right), n=1, \cdots, s, l=1, \cdots, s^{\prime}$. The CCD is equal to $k s^{\prime}+s k^{\prime}$. For wavefunctions of the relative part in $F\left(z_{i}\right)$ (denoted as $F^{\prime}$ here), one can show that $F^{\prime}$ of the bosonic model and $F_{b o}^{\prime}$ of the anyon model satisfy the same translational properties and have the same dependence on holomorphic coordinates. Thus the possible $F^{\prime}$ are given by $s^{\prime}$-dimensional vectors $F_{l}^{\prime}$ with $l=1, \cdots, s^{\prime}$, similar to the case of the bosonic model. By the same reasoning as for the bosonic model, we can expand $F$ as $\sum_{l} \theta\left[\begin{array}{c}a_{m, n, l} \\ b\end{array}\right]\left(\sum_{i} z_{i} e_{2} \mid e_{1}, \tau\right) F_{l}^{\prime}$. The final wavefunctions have $s$-components and the component index is $n$.

Since the vectors $F_{l}^{\prime}$ satisfy the same translational properties as the vectors $F_{l}^{\prime b o}$ and depend only on holomorphic coordinates, the number of possible $F_{l}^{\prime}$ is equal to the number of possible $F_{l}^{\prime b o}$. Let $D$ be the DHMS of the anyon model and $D_{b o}$ be that of the bosonic model. As the wavefunctions of the relative part for both models have the same degeneracy, $D$ divided by $D_{b o}$ is equal to the CCD of the anyon model divided by that of the bosonic model. So $D / D_{b o}=\left(k s^{\prime}+s k^{\prime}\right) / k^{\prime}$. Since $D_{b o}=\left(\Phi^{\prime}+N-1\right) ! /\left[\left(\Phi^{\prime}-1\right) !(N) !\right], D$ is given by Eq. (6), obtained previously for a particular $\Phi^{\prime}$.

The problems discussed in this letter can be formulated equivalently in the context of Chern-Simons theory. In 24 the complete set of LLL wavefunctions were written down for bosons interacting with both a statistics transmuting Chern-Simons field and an external magnetic field. After a suitable gauge transformation the wavefunctions were shown to be multivalued and multicomponent consistent with braid group arguments. Thus, this is a model for anyons in an external magnetic field.
We will follow closely Ref. 24] (We note that the notation in Ref. 24] is different from that used here). Each wavefunction is specified by a $N$ component vector of integers $\mathbf{c}$ and an integer $0 \leq K<\operatorname{gcd}\left(\Phi^{\prime}, N\right)(\operatorname{gcd}$ is the greatest common divisor of two numbers). The components of $\mathbf{c}$ are ordered $0 \leq c_{1} \leq c_{2} \ldots \leq c_{N}<$ $s \Phi^{\prime}\left(s \Phi^{\prime}+k N\right) \equiv \ell$ and satisfy a constraint:

$$
c_{i}+\frac{k}{s \Phi^{\prime}} \sum_{j=1}^{N} c_{j}=\left(s \Phi^{\prime}+k N\right)\left(m_{0}+s m_{i}\right)
$$

for some integers $m_{0},\left\{m_{i}\right\}$ with $0 \leq m_{0}<s$. In addition, we require that the first component of the vector $\mathbf{c}, c_{1}$, be strictly less than $\ell / \operatorname{gcd}\left(N, \Phi^{\prime}\right)$. Given a solution $\mathbf{c}$ to the above constraint (7), we define a new integer $M$ as the smallest integer such that the action $c_{i} \rightarrow c_{i}+M \frac{\ell}{\operatorname{gcd}\left(\Phi^{\prime}, N\right)}$ results in a permutation of the components of $\mathbf{c}$ with the components defined $\bmod \ell$. Then $K$ takes $M$ values: $n \operatorname{gcd}\left(\Phi^{\prime}, N\right) / M, n=0, \ldots, M-1$.

To count the DHMS (denoted also as $D$ ) in the LLL we need to count all the vectors $\mathbf{c}$ satisfying the constraint (7) for a given $M$, let us call this number $d(M)$. Then the DHMS is given by $D=\sum_{M=1}^{\operatorname{gcd}\left(\Phi^{\prime}, N\right)} M d(M)$. The $M$ multiplying $d(M)$ is due to the $M$ values taken by $K$. We notice that $D$ can be equivalently calculated as the total number of c's satisfying the constraint (7) with $0 \leq c_{1} \leq$ $c_{2} \ldots \leq c_{N}<s \Phi^{\prime}\left(s \Phi^{\prime}+k N\right) \equiv \ell$ with no further restriction on the range of $c_{1}$. This simplifies the problem considerably. It immediately follows that the most general form for $\mathbf{c}$ is given by: $c_{i}=s\left(c_{0}+\left(s \Phi^{\prime}+k N\right) n_{i}\right)$, with $0 \leq n_{1} \leq n_{2} \leq \ldots \leq n_{N}<\Phi^{\prime}, 0 \leq c_{0}<\left(s \Phi^{\prime}+k N\right)$ and the condition that

$$
\frac{c_{0}+k \sum_{i=1}^{N} n_{i}}{\Phi^{\prime}} \in Z
$$

The number of distinct vectors $\mathbf{c}$ is given by the total number of values that $c_{0}$ can take times the total number of integers $0 \leq n_{1} \leq \ldots \leq n_{N}<\Phi^{\prime}$. The number of $c_{0}$ is simply $\left(s \Phi^{\prime}+k N\right)$, while the total number of $n_{i}$ is given by the standard combinatoric result $\left(\Phi^{\prime}+N-1\right) ! /\left[\left(\Phi^{\prime}-1\right) ! N !\right]$. Thus the total number of vectors $\mathbf{c}$ is given by

$$
\left(s \Phi^{\prime}+k N\right) \frac{\left(\Phi^{\prime}+N-1\right) !}{\left(\Phi^{\prime}-1\right) ! N !} .
$$

How many of these vectors satisfy the condition (8)? If $k N$ is divisible by $\Phi^{\prime}$ then for every given set of integers $\left\{n_{i}\right\}$, we can find a sequence containing $\left(k N+s \Phi^{\prime}\right) / \Phi^{\prime}$ values of $c_{0}$ separated by a multiple of $\Phi^{\prime}$ such that condition (8) is fulfilled. Therefore, $D$, the total degeneracy of LLL states, is given by (9) divided by $\Phi^{\prime}$, which leads to our previous formula (6) for the DHMS of LLL states. In cases where $k N$ is not divisible by $\Phi^{\prime}$ we have failed to find a rigorous proof of formula (6), however, we have 
checked it for a number of potentially anomalous cases and found that it gives the correct degeneracy.

The formula (6) for the DHMS is sensible for a number of cases. Even though we have implicitly assumed that $\Phi^{\prime} \neq 0$ the formula gives a sensible answer even for $\Phi^{\prime}=0$, namely $D=k$ which agrees with the result of 24]. For the fermionic $(k=s=1)$ and bosonic $(k=$ $0, s=1$ ) cases the standard degeneracy is reproduced. Following Ref. [9], we also calculated the thermodynamics on a torus by using the formula of the DHMS and we found that the thermodynamics is the same as that on a sphere (see also Ref. 8]). To compare Eq. (6) with Eq. (2), we write $D$ as $D_{1}=\left(\Phi^{\prime}+N-1\right) ! /\left[\left(\Phi^{\prime}-1\right) ! N !\right]$ times $D_{2}=(s \Phi \prime+k N) / \Phi^{\prime}$, where $\Phi^{\prime}=\Phi-\alpha N$ corresponds to $d$ and $\alpha$ corresponds to $g$ in Eq. (1) and Eq. (2). $D_{1}$ is Haldane's 'choose' expression (Eq. (2)) and $D_{2}$ is the correction due to the center coordinate degeneracy.

We now apply the above results to the FQHE. In the case of QH excitations of a Laughlin state at filling $1 / \mathrm{m}$, the effective flux $\Phi^{\prime}$ for the quasiparticle, the sum of the magnetic flux and the statistical flux is equal to the number of electrons $N_{e}$ in the parent state. The statistical parameter $\alpha$ is $1 / m$. Let $N_{q}$ be the number of quasiholes, we apply formula (6) to the current case, the DHMS of the low-energy spectrum is $\frac{\left(N_{e}+N_{q}-1\right) !}{\left(N_{e}\right) !\left(N_{q}\right) !}\left(m N_{e}+N_{q}\right)$.

The DHMS in the case of QE excitations in a Laughlin state can also be worked out. The statistical flux and the magnetic flux are now parallel, and it is important to use hard-core boundary conditions as in Refs. 11 13 to obtain the following formula. One part of the wavefunction is now $\prod_{i<j}\left[\theta_{3}\left(z_{i}-z_{j} \mid \tau\right)\right]^{-1 / m} F\left(z_{i}\right)_{n}$. However because of hard-core boundary conditions, $F\left(z_{i}\right)_{n}$ should be factorized as $\prod_{i<j}\left[\theta_{3}\left(z_{i}-z_{j} \mid \tau\right)\right]^{2} F_{q}$. We note $F\left(z_{i}\right)_{n}$ and $F_{q}\left(z_{i}\right)$ have the same CCD. One can then derive the number of solutions of $F_{q}\left(z_{i}\right)$ (following the methods used previously), which is the DHMS in the case of QE excitations: $\frac{\left(N_{e}-N_{q}-1\right) !}{\left(N_{e}-2 N_{q}\right) !\left(N_{q}\right) !}\left(m N_{e}-N_{q}\right)$.

When $N_{q}=0$, the formulas of the DHMS in both cases are equal to $m$, which is the degeneracy of the Laughlin state on the torus. $m$ is the CCD of the Laughlin state, which was investigated in detail by Haldane (the third reference in Ref. 20]).

The details of the derivations of this paper and the formula in the case of multispecies anyons with mutual statistics on a torus will be presented elsewhere.

DL would like to thank Prof. S. Ouvry and Prof. P. Sodano for stimulating discussions and encouragement, and Prof. R. Iengo for discussions on anyon physics over the last several years. He also thanks CNRS for the supports of his stay at IPN, Orsay where the work started. AF would like to thank A. Karlhede for an enlightening discussion on thermodynamics of anyons.
[1] J.M. Leinaas and J. Myrheim, Nuovo Cimento B 37, 1 (1977); G.A. Goldin, R. Menikoff and D.H. Sharp, J. Math. Phys. 22, 1664 (1981); F. Wilczek, Phys. Rev. Lett. 48, 1144 (1982); 49, 957 (1982); Y.-S. Wu, ibid. 52, 2103 (1984); 53, 111 (1984).

[2] R. Laughlin, Phys. Rev. Lett. 50, 1395 (1983).

[3] R. Prange and S. Girvin, The Quantum Hall Effect (Springer-Verlag, New York, Heidelberg, 1990, 2nd ed), and references therein.

[4] B.I. Halperin, Phys. Rev. Lett. 52, 1583 (1984).

[5] D. Arovas, J.R. Schrieffer and F. Wilczek, Phys. Rev. Lett. 53, 722 (1984).

[6] F.D.M. Haldane, Phys. Rev. Lett. 51, 605 (1983).

[7] F.D.M. Haldane, Phys. Rev. Lett. 67, 937 (1991).

[8] A. Dasnières de Veigy and S. Ouvry, Phys. Rev. Lett. 72, 600 (1994).

[9] Y.-S. Wu, Phys. Rev. Lett. 73, 922 (1994).

[10] S.B. Isakov, Mod. Phys. Lett. B 8, 319 (1994); Int. J. Mod. Phys. A 9, 2563 (1994); D. Bernard and Y.-S. Wu, cond-mat/9404025; F. Lesage, V. Pasquier and D. Serban, Nucl. Phys. B 435, 585 (1995); Z.N.C. Ha, Phys. Rev. Lett. 73, 1574 (1994); Nucl. Phys. B 435, 604 (1995); A. Dasnières de Veigy and S. Ouvry, Mod. Phys. Lett. B 9, 271 (1995), and references therein.

[11] S. He, X.C. Xie, and F.C. Zhang, Phys. Rev. Lett. 68, 3460 (1992); M. Ma and F.C. Zhang, ibid. 66, 1769 (1991); J. Yang and W.P. Su, ibid. 68, 2382 (1992); ibid. 70, 1163 (1993); Phys. Rev. B 47, 12953 (1993).

[12] M.D. Johnson and G.S. Canright, J. Phys. A 27, 3579 (1994); Phys. Rev. B 49, 2947 (1994).

[13] D. Li and S. Ouvry, Nucl. Phys. B 430, 563 (1994) (FS).

[14] T. Einarrson, Phys. Rev. Lett. 64, 1995 (1990); Mod. Phys. Lett. B 5, 675 (1991), and references therein.

[15] D. Li, Mod. Phys. Lett. B 7, 1103 (1993).

[16] R. Iengo and K. Lechner, Phys. Rep. C 213, 179 (1992), and references therein; Nucl. Phys. B 346, 551 (1990); ibid. 365, 551 (1991).

[17] A. Fayyazuddin, Int. J. Mod. Phys. A 21, 3785 (1994).

[18] Y. Hosotani and C-L Ho, in "the Proceedings of the 26th ICHEP", and references therein.

[19] J.K. Jain, Phys. Rev. Lett. 63, 199 (1989); Phys. Rev. B 41, 7653 (1990); Adv. phys. 41, 105 (1992); G. Dev and J.K. Jain, Phys. Rev. Lett. 69, 2843 (1992).

[20] R. Tao and Y.-S. Wu, Phys. Rev. B 30, 1097 (1984); R. Tao and F.D.M. Haldane, ibid. 33, 3844 (1986); F.D.M. Haldane, Phys. Rev. Lett. 55, 2095 (1985); F.D.M. Haldane and E.H. Rezayi, Phys. Rev. B 31, 2529 (1985).

[21] W.P. Su, Phys. Rev. B 30, 1069 (1984).

[22] D. Li, Int. J. Mod. Phys. B 7, 2779 (1993); ibid. 7, 2655 (1993).

[23] The second Ref. in Refs. 20.

[24] A. Fayyazuddin, Mod. Phys. Lett. A 8, 3173 (1993). 